\section{La técnica de osteotomo en implantología oral}

Piaggio-Bravo L, Delgado-Bravo M, Ccahuana-Vásquez V, Alarcón-Palacios MA. La técnica de osteotomo en implantología oral. Rev Estomatol Herediana. 2011; 21(1):38-43.

RESUMEN

Se sabe que luego de una extracción dentaria el proceso alveolar esta sujeto a continuo remodelado óseo, así mismo, con el incremento de la edad los procesos de reabsorción predominan sobre la nueva formación ósea, así la perdida de dientes da lugar a un incremento en la reabsorción del proceso alveolar y a cambios en su morfología. Con el paso de los años, diferentes sistemas de implantes han sido introducidos con una sustancial variación en el sistema de fresado o perforación, así como también en la técnica quirúrgica aplicada. Obtener estabilidad inicial durante la colocación de los implantes es en efecto, uno de los criterios fundamentales para obtener oseointegración y lograr esta estabilidad depende de la densidad ósea, la técnica quirúrgica utilizada y las características macroscópicas y microscópicas del implante utilizado. Así cuando existen deficiencias en el volumen del tejido óseo fundamentalmente en ancho y este no cumple con los requisitos mínimos exigidos para la obtención de estabilidad, las técnicas de expansión crestal deben ser tomadas en cuenta para la ubicación de los implantes con predicibilidad.

Palabras clave: IMPLANTES DENTALES / OSTEOTOMO / EXPANSIÓN ÓSEA.

Osteotome techinque in oral implantology

ABSTRACT

It is known that after a tooth extraction the alveolar process is subject to continuous bone remodeling. Also, with aging, resorption processes predominate over the new bone formation and tooth loss leads to an increase in the resorption of the alveolar process and changes in its morphology. Over the years, many different implant systems have been introduced with a substantial variation in the drilling system as well as the surgical technique applied. To get initial implant stability is indeed one of the fundamental criteria for obtaining osseointegration and achieve this stability depends on bone density, the surgical technique used and the macroscopic and microscopic characteristics of the implant used. Therefore, when there are deficiencies in the volume of bone tissue mainly in width and this does not meet the minimum requirements for obtaining stability, crestal expansion techniques should be taken into account for the location of the implants with predictability.

Key words: DENTAL IMPLANTS / OSTEOTOME / BONE EXPANSION.
Luis Alejandro Piaggio Bravo Miguel S. Delgado Bravo² Vanessa Ccahuana Vásquez ${ }^{1}$ Marco A. Alarcón Palacios²

'Docente del Departamento Académico de Clínica Estomatológica

${ }^{2}$ Docente del Departamento Académico de Medicina y Cirugía Buco Maxilofacial.

Facultad de Estomatología. Universidad Peruana

Cayetano Heredia

\section{Correspondencia}

Luis Alejandro Piaggio Bravo La Floresta 365, Lima 41 - Perú Teléfono: 3722458

e-mail: luis.piaggio@upch.pe

Recibido : 16 de enero de 2011

Aceptado : 20 de marzo de 2011

\section{Introducción}

El mantenimiento de los rebordes alveolares después de la pérdida de dientes ha significado un constate problema durante muchos años en la odontología. Los métodos clásicos de rehabilitación protésica, se han basado en la colocación de injertos de diverso origen como hueso autógeno o sustitutos óseos inertes con la finalidad de conseguir que retrase el proceso de reabsorción ósea alveolar(1).

Es sabido que la tasa de éxito obtenida con implantes dentales en diversas situaciones clínicas depende en gran medida de la calidad de la unión implante/hueso, el volumen y la calidad del hueso que lo rodea. La estabilidad inicial de los implantes es uno de los criterios fundamentales para obtener oseointegración, sin embargo, lograr una óptima unión implante hueso depende también de la técnica quirúrgica utilizada, las características del hueso periimplantario y de las características macroscópicas y microscópicas del implante utilizado $(2,3)$.

En la última década las técnicas de colocación de implante han evolucionado rápidamente, lo que ha permitido afrontar mejor los casos en los que antes no era aconsejada la colocación de implantes. Cuando el ancho óseo no cumple los requisitos mínimos exigidos, las técnicas de expansión crestal son indispensables para la ubicación de los implantes con buena predicibilidad (4).

El propósito del presente trabajo es presentar una revisión de la literatura científica relacionada con la aplicación de la técnica de osteotomo para la colocación de implantes dentales.

\section{Revisión de la literatura}

Desde que la técnica de osteótomo transalveolar para elevación del piso de seno maxilar fue introducida en 1986, varios estudios han hecho reportes sobre las ventajas de esta técnica (5-11). En la publicación original de Tatum en 1986 (10), un instrumento especial conocido como formador de alveolo fue usado para fracturar el piso de seno maxilar y moverlo en una dirección más apical. Al comienzo el autor no utilizó ningúnmaterial de injerto para incrementar y mantener el volumen del área elevada. Posteriormente Summers en 1994 (12) describió otra técnica transalveolar para la elevación de seno maxilar utilizando osteotomos y adicionando hueso. Es este autor quien con más aciertos diseñó instrumentos conocidos como osteótomos. Estos instrumentos de forma cilindro-cónica van aumentando su grosor pro- 
gresivamente con el propósito de expandir y compactar las trabéculas óseas, empujándolas en una dirección vertical y elevando también la membrana sinusal. De esta forma, se va aumentando la densidad ósea y la superficie de fricción. Los osteótomos de punta fina se utilizan en aquellos casos en que existe hueso tipo II ó III según la clasificación de Lekholm y Zarb (1985), en los que no se necesita conseguir una compactación sino una expansión debido a la estrechez ósea como son las crestas en filo de cuchillo $(5,6)$.

\section{Concepto de osteodilatación}

La osteodilatación consiste en expandir las crestas óseas estrechas con el fin de conseguir un ancho de hueso aceptable para la colocación de los implantes (7). La osteodilatación o expansión ósea también puede ser definida como la manipulación del hueso para formar un lecho receptor que permita colocar un implante sin la remoción de hueso del paciente y cuyo objetivo es mantener el hueso existente a través de empujar la tabla ósea bucal del reborde residual con mínimo trauma (8). La técnica de osteótomos aplica el concepto de osteodilatación esta fue introducida para incrementar la estabilidad primaria de los implantes dentales en la maxila posterior, así este procedimiento ha sido bien establecido en la práctica clínica de manera rutinaria (2). Los procedimientos de ampliación y expansión fueron inicialmente desarrollados por Tatum en 1986 como un método alternativo para la colocación inmediata de los implantes en rebordes residuales atróficos $(9,10)$. Summers en 1994, presentó los primeros osteodilatadores con forma cilindrocónica y con diámetros que aumentan progresivamente de un instru- mento a otro, de tal modo que la base de cada uno de ellos se corresponde con la parte activa del siguiente. Esto permite introducirlos en el hueso y comprimir las trabéculas, consiguiendo una mayor densidad ósea para preparar lechos con diámetro similar al implante requerido. La parte apical del instrumento es cóncava y existen seis diámetros diferentes marcados a distintas longitudes.

Esta técnica ha modificado el procedimiento quirúrgico implantológico en el maxilar superior por la existencia de un hueso más esponjoso y las características anatómicas del mismo y posterior a su aparición se han venido utilizando diversas variantes de los osteótomos, generalmente en combinación con cinceles, tornillos de expansión u otras técnicas quirúrgicas, pero que han permitido siempre la colocación de los implantes en la misma intervención quirúrgica. Con el paso del tiempo los osteótomos han sido aplicados en la técnica de angulación parasinusal, elevación directa e indirecta del seno maxilar y colocación de implantes en la zona pterigoidea. La revisión de la literatura científica de implantes orales revelan que la técnica de osteótomo ha sido generalmente llevada a cabo en combinación con la elevación del piso del seno maxilar (2,3,7,10-13).

En la actualidad existen diversos modelos de osteodilatadores basados en los que describió Summers, los que incorporan algunas variaciones como el diseño del extremo apical o diferentes calibres, que se adaptan a los diversos sistemas de implantes. También existen osteótomos roscados, tanto para la maxila como para la mandíbula, así como angulados que permitien un mejor acceso en los segmentos pos- teriores (7).

\section{Indicaciones y contraindicacio- nes}

Se han propuesto las siguientes técnicas de expansión ósea; con el objetivo de conseguir mayor volumen de hueso:

1.Expansión ósea con osteótomos o expansores roscados.

2.Expansión ósea con corticotomía crestal.

3.Expansión ósea con corticotomía crestal y una o dos descargas laterales.

Como norma general, se indica la técnica de expansión ósea para aumentar el ancho de la cresta alveolar cuando esta es inferior a los $6 \mathrm{~mm}$. En el maxilar superior, la técnica de expansión con osteótomos, permite aumentar el volumen óseo en $4 \mathrm{~mm}$. Sin embargo en la mandíbula esta ganancia ósea queda reducida a un máximo de $1,5 \mathrm{~mm}$. De la misma forma, los expansores roscados permiten conseguir los mismos resultados. Este aumento de anchura servirá para conseguir volumen de hueso suficiente para el alojamiento del implante con mejor predictibilidad (4).

En muchas ocasiones las técnicas de expansión con osteótomos son insuficientes, para lograr un volumen mínimo de hueso receptor, por lo que en estos casos la utilización de corticotomías, permite desplazar las corticales óseas. También permite corregir los defectos anatómicos vestibulares que suelen acompañar a la reabsorción de la cresta ósea. La reabsorción del hueso de la maxila se caracteriza por ser centrípeta, reduciéndose en gran medida la cortical vestibular, con lo cual en el caso de colocar los implantes en el hueso maxilar residual, nos encontramos ante una discrepancia importante si queremos que 
el anclaje oclusal y el perfil de emergencia sea el adecuado (4).

La expansión sin corticotomía estará indicada en aquellos casos leves en que se precisa una ganancia media de $3 \mathrm{~mm}$, con hueso no cortical y formas triangulares. La expansión con corticotomías se puede realizar en aquellos casos en los que se requiera un mayor aumento del ancho óseo, de tal manera que el aumento de la neovascularización y sangrado provocado por el corte, beneficie a los procesos de neoformación ósea, aunque principalmente está indicada en aquellos casos con crestas óseas de 3 a $6 \mathrm{~mm}$ de ancho (4). La presencia de crestas alveolares atróficas con menos de 3mm de ancho dificulta la colocación de implantes y hace necesaria la utilización complementaria de injertos óseos (7).

La posibilidad de anclar los implantes en la cortical interna de los senos maxilares o de las fosas nasales, asegura una buena estabilidad primaria de los mismos, es lo que llamamos anclaje bicortical, el que se consigue a través del tacto del operador y que es proporcionado con la utilización manual de los osteótomos frente a las fresas quirúrgicas (7).

En las elevaciones del piso del seno maxilar, cuando se realiza un abordaje directo, se despega y se eleva la mucosa sinusal, se rellena con un material de injerto autólogo o heterólogo y se colocan los implantes en una segunda intervención quirúrgica o en la misma, en función del hueso remanente que exista para conseguir una adecuada fijación primaria. En estos casos, el uso de los osteodilatadores para crear el lecho está indicado como una técnica segura que ahorra hueso y facilita la oseointegración de los implantes (7).

Hay situaciones que merecen es- pecial atención y cuidado, no porque la técnica este contraindicada, sino por la dificultad de movilizar el fragmento desplazado, sin perder la conexión ósea directa, estas son: cretas maxilares con un reborde estrecho, un perfil rectangular, paredes paralelas que dificultan la separación del colgajo óseo y con depresión vestibular en la parte apical del mismo lo cual aumenta el riesgo de ruptura y desprendimiento del fragmento óseo; crestas mandibulares estrechas, que suelen tener el perfil triangular y que por la gran corticalización del colgajo óseo se vuelvan quebradizas y poco elásticas (4).

\section{Técnica quirúrgica}

La técnica básica consiste en ampliar el lecho implantario utilizando de forma progresiva los osteótomos hasta conseguir la expansión deseada, sin embargo la técnica varia según las necesidades de la expansión ósea. En los casos donde no se requiera corticotomía crestal, en primer lugar se perforará la cortical alveolar con una fresa redonda o lanza para introducir el instrumento más fino y continuar con los osteotomos de mayores diámetros. Los osteótomos se introducen manualmente, presionando y rotando al mismo tiempo, hasta alcanzar la altura deseada o sentir resistencia. En ese caso, se puede percutir suavemente con un martillo quirúrgico, recurrir a instrumentos rotatorios pasando la fresa piloto de $2 \mathrm{~mm}$ de diámetro o incluso alternando fresas y osteótomos de menor a mayor tamaño. Una vez alcanzada la profundidad deseada y antes de pasar al siguiente instrumento, es aconsejable esperar entre 30 y 40 segundos, para que las microfracturas óseas producidas vayan dilatando y compactando el hueso adyacente. Conseguido el lecho se insertará el implante inmediatamente para evitar que el alveolo se colapse y sólo se pasará el macho de terraja en aquellos casos en los que la densidad ósea sea elevada. La colocación del implante deberá ser especialmente cuidadosa para evitar dehiscencias o fracturas de la tabla vestibular, que en ocasiones puede encontrarse muy adelgazada. Las fracturas verticales de la cortical vestibular, siempre que no comprometan la estabilidad primaria del implante, no tienen ninguna trascendencia puesto que consolidan durante la fase de cicatrización $(4,7)$.

En los casos donde se realice una corticotomía crestal, se realizará la misma incisión supracrestal y elevación del colgajo a grosor completo, mucho más amplio para permitir el paso de los discos de corte óseo sin lesionar los tejidos blandos. Se insinúa el fresado óseo con la fresa lanza allí donde se colocarán los implantes. A continuación se procederá a marcar ligeramente la cresta con el disco de menor diámetro hasta obtener un surco de $0,3 \mathrm{~mm}$ de anchura y $1 \mathrm{~mm}$ de profundidad, sobrepasando los puntos marcados con la fresa lanza para la colocación de los implantes permitiendo así, según la elasticidad ósea, la separación de la tabla vestibular y palatino o lingual sin roturas. Posteriormente se pasará progresivamente los discos de mayor diámetro. Todos los pasos deben realizarse con refrigeración suficiente, para evitar el recalentamiento óseo y a velocidad de 20000 rpm (4,7).

A continuación, se colocará parcialmente los expansores roscados hasta conseguir una expansión parcial que se mantendrá con los propios instrumentos o expansores en el interior de las dos paredes óseas 
en la zona crestal, lo que permitirá mantener el máximo grosor de pared ósea. Una vez conseguido el eje y la longitud deseada, se colocará los expansores con lentitud hasta llegar a la profundidad adecuada, dejando al hueso tiempo suficiente para que se adapte a la deformación en lugar de fracturarse. Acto seguido, se retirará el osteótomo con menos tensión y se sustituirá por el implante seleccionado $(4,7)$.

Cuando la elasticidad ósea no es suficiente, o el espacio a expandir esté limitado, se procede como los casos anteriores a realizar una incisión crestal, en este caso más amplia, con dos incisiones laterales de descarga, de tal manera que sobrepasen la zona a expandir en $6 \mathrm{~mm}$ y aparte de realizar el fresado crestal con discos se realizan uno o dos fresados o cortes transversales de descarga, que serán más largos cuanto más importante sea la expansión a realizar. Estas corticotomías transversales de descarga deben de tener una ligera inclinación que permita la vestibulización de la porción ósea y a su vez cruzarse con la corticotomía crestal, para que la abertura sea lo más suave y correcta posible. En los ángulos formados está indicado utilizar cinceles de corte afilado y de una achura media de $3 \mathrm{~mm}$. A continuación, se utilizan los osteótomos roscados como en el caso precedente y se insertan los implantes $(4,7)$.

\section{Discusión}

Los implantes dentales han ampliado las opciones protésicas y actual mente se han convertido en instrumentos importantes para la rehabilitación del edéntulo en el sector anterior y posterior. Ellos proporcionan una plataforma estable desde la cual se pueden construir dispositivos removibles y fijos. Mientras que la ubicación de un implante en la mandíbula presenta pocas limitaciones quirúrgicas, excepto por la presencia del nervio dentario inferior, en el sector posterior de la maxila se presenta una serie de dilemas quirúrgicos, tales como la calidad ósea (hueso tipo III ó IV), cortical delgada o nula, insuficiente altura ósea etc (14).

Desde que Summer (12) introdujo su técnica con osteótomos en 1994, esta se ha ido incorporando dentro de la práctica odontológica con buenos resultados clínicos. El uso de osteótomos es una técnica relativamente sencilla, que aplicada por un profesional con suficiente experiencia y realizada cuidadosamente, presenta pocas complicaciones y permite la inserción de los implantes en la misma sesión $(12,15)$. Dicho procedimiento es una excelente solución en aquellos casos en que la estrechez ósea alveolar no nos permite insertar implantes, teniendo que recurrir a técnicas de regeneración ósea que resultaban en tratamientos complicados e incómodos para el paciente (1).

Komarnychyj y London (16) (1988), colocaron 43 implantes en sitios individuales preparados con la técnica de osteotomo, 41 de ellos fueron exitosamente integrados en un intervalo de tiempo de 9 meses hasta aproximadamente 4 años, estos 41 implantes representaron un tasa de éxito del 95,3\%. Todos los implantes fueron restaurados en función completa en un intervalo entre tres y 38 meses, ningún implante fracasó luego de la restauración final.

Bruschi et al. (17) (1998), trataron 303 pacientes a los cuales se les colocaron 499 implantes usando la técnica de manejo localizado del piso del seno maxilar (LMSF) entre abril de 1988 y diciembre de 1993, para la confección del lecho de los implantes se utilizó expansores con diámetro diferente. Los pacientes seleccionados no mostraron signos de patología sinusal, tenian insuficiente reborde alveolar en sentido vertical para la colocación de implantes y presentaron una altura ósea residual la cual estuvo entre 5 y $7 \mathrm{~mm}$. La tasa de éxito obtenida para los 499 implantes colocados con esta técnica fue de 97,5\%.

Rosen et al. (18) (1999), realizaron un estudio multicéntrico, donde evaluaron 174 implantes colocados en 101 pacientes, en el tratamiento de estos pacientes se utilizó la elevación del piso de seno maxilar con osteótomos adicionando hueso, en el estudio participaron nueve clínicos, los cuales usaron distintos injertos óseos, el promedio de periodo de carga del implante fue de 20,2 meses con un rango de 6 a 66 meses, encontrando una tasa de supervivencia de $95,4 \%$.

Vilaplana et al. (1) (2000) realizaron un estudio donde presentaron una serie de 18 pacientes a los cuales se le insertaron 40 implantes Steri-oss cilíndricos recubiertos con hidroxihiapatita entre los años 95 y 98. Dos de los implantes se perdieron al inicio de la fase protésica, no hubo rechazo pero su estabilidad no era totalmente correcta procediendo al retiro de los implantes. Los implantes correspondieron a un incisivo superior derecho y un segundo premolar superior izquierdo en diferentes pacientes. Ellos concluyen que la técnica de expansión ósea permite la inserción de implantes en crestas alveolares estrechas y la compactación de hueso esponjoso incrementando su fricción.

Emmirech et al. (19) (2005), realizarón un meta-análisis sobre 1139 implantes colocados con la técnica de osteótomos, obteniendo un 
tasa de supervivencia del 97,5\% y $90,9 \%$ después de 12 y 36 meses de cargados, respectivamente.

Shalabi et al. (9) (2007), Realizaron un meta- análisis cuyo propósito fue estimar la tasa de supervivencia para implantes colocados con la técnica de osteótomos. La información obtenida de 349 implantes reveló tasas de supervivencia del $98 \%$ hasta en inicio de la carga y de $99 \%$ después de 56 meses de carga funcional, concluyendo que el resultado obtenido usando la técnica de osteótomo en relación a la supervivencia de los implantes pareció ser similar a la ubicación de implantes por medio de la técnica convencional.

Sforza et al. (20) (2008), realizaron un estudio cuyo objetivo fue describir y evaluar una técnica de aumento del piso maxilar modificada usando la técnica de osteotomo simplificado. Se trataron 26 pacientes con 39 implantes Branemark (Nobel Biocare) con implantes de longitud de 10 a $15 \mathrm{~mm}$. El tiempo desde la instalación de la prótesis hasta su último control tuvo una variación de 5 a 74 meses. Sólo se registró la falla de un implante lográndose una taza de éxito del $94,7 \%$ concluyendo que los datos obtenidos junto con la ausencia de complicaciones sugieren que la técnica de osteótomo simplificado puede ser considerada efectiva y segura aunque son necesarios estudios a largo plazo .

Velasco et al. (15) (2008), Llevaron a cabo un estudio cuyo objetivo fue realizar una valoración clínica y seguimiento del tratamiento de pacientes con implantes oseointegrados insertados mediante la técnica de expansión ósea. Se trataron 56 pacientes a los cuales se les colocaron 117 implantes microdent, 106 implantes fueron colocados en la maxila $(90,5 \%)$ y 11 implantes en la mandíbula (9,5\%), solo dos implantes presentaron complicaciones y se perdieron durante el periodo de cicatrización, ningún implante se perdió después de la carga funcional en un periodo de seguimiento de 14,1 meses obteniéndose una tasa de éxito de $98,3 \%$. Se concluyó que el tratamiento con implantes mediante la técnica de expansión ósea constituye una alternativa terapéutica implantológica de éxito.

Nedir et al. (21) (2009), realizaron dos reportes de caso de colocación de implantes cónicos (8 y 10mm. de longitud), utilizando la técnica de elevación de piso de seno en zonas atróficas posteriores mediante osteótomos, sin ningún material de injerto, demostrando que en aquellos casos donde sólo se podía considerar una ventana lateral, se pudo realizar esta técnica de manera menos invasiva y de corto procedimiento. Todos los implantes lograron estabilidad primaria y fueron cargados satisfactoriamente después de 3,6 meses y a los posteriores controles en el primer y segundo año, estos implantes con sus prótesis definitivas se mantenían estables y en funcionamiento.

\section{Conclusiones}

En base a la revisión bibliográfica que respalda la utilización de la técnica de osteótomo para la colocación de implantes, se puede concluir que esta técnica nos ofrece los siguientes beneficios:

- Permite la colocación inmediata de implantes dentales en rebordes alveolares estrechos y es una alternativa viable a la colocación de injertos en bloque de tejido óseo en casos previamente seleccionados.

- Se recomienda su utilización apli- cando una fuerza controlada y gradual y esperar esperar entre los diferentes diámetros de osteótomos entre 40 segundos y 1 minuto.

- Puede ser usada en ambos maxilares, pero se encuentra indicada en casos donde exista hueso tipo III o tipo IV.

- La técnica permite expandir, compactar y elevar la membrana sinusal y puede ser utilizada con la mayoría de diseños de implante.

- La técnica tiene poca morbilidad, es mínimamente invasiva y provee excelentes resultados, aún para cirujanos poco diestros.

\section{Referencias bibliográficas}

1. Vilaplana Gómez JA, Méndez Trujillo S, Ortega López J.J, Vilaplana Vivo J. Técnica de los osteotomos en implantología. 2000; 12(1):43-8.

2. Büchter A, Kleinheinz J, Wiesmann HP, Jayaranan M, Joos U, Meyer U. Interface reaction at dental implants inserted in condensed bone. Clin Oral Implants Res. 2005; 16(5):509-17.

3. Büchter A, Kleinheinz J, Wiesmann HP, Kersken J, Nienkemper M, Weyhrother H, Joos U, Meyer U. Biological and biomechanical evaluation of bone remodelling and implant stability after using an osteotome technique. Clin Oral Implants Res. 2005; 16(1):1-8.

4. Padros A. Pedemonte E. Padulles E. Benet O. Arano J. Tecnica de expansión de cresta estrecha. Rev Esp Odontoestomatol Implant. 2005; 13(1):7-12.

5. Pjetursson BE, Ignjatovic D, Matuliene G, Brägger U, Schmidlin K, Lang NP. Transalveolar maxillary sinus 
floor elevation using osteotomes with or without grafting material. Part II: Radiographic tissue remodeling. Clin Oral Implants Res. 2009; 20(7):677-83.

6. Vilaplana Gómez JA, Méndez Trujillo S, Ortega López JJ, Vilaplana Vivo J. Técnica de los osteotómos en implantología. Avances. 2000; 12(1): 43-8.

7. Rambla-Ferrer J, PeñarrochaDiago M, Guarinos-Carbó J. Analysis of the use of expansion osteotomes for the creation of implant beds. Technical contributions and review of the literature. Med Oral Patol Oral Cir Bucal. 2006; 11(3):E267-71.

8. Goyal S, Iyer S. Bone Manipulation Techniques. International Journal of Clinical Implant Dentistry [serial on the Internet]. (2009, Jan), [cited September 29, 2010]; 1(1): 2231. Available from: Dentistry \& Oral Sciences Source

9. Shalabi MM, Manders P, Mulder J, Jansen JA, Creugers NH. A meta-analysis of clinical studies to estimate the 4.5-year survival rate of implants placed with the osteotome technique. Int J Oral Maxillofac Implants. 2007; 22(1):110-6.

10. Tatum H Jr. Maxillary and sinus implant reconstructions. Dent Clin North Am. 1986; 30(2):20729.

11. Blanco J, Suárez J, Novio S,
Villaverde G, Ramos I, Segade

LA. Histomorphometric assessment in human cadavers of the peri-implant bone density in maxillary tuberosity following implant placement using osteotome and conventional techniques. Clin Oral Implants Res. 2008; 19(5):505-10.

12. Summers RB. A new concept in maxillary implant surgery: the osteotome technique. Compendium. 1994 Feb;15(2):152-8.

13.Santagata M, Guariniello L, D'Andrea A, Tartaro G. A modified crestal ridge expansion technique for immediate placement of implants: a report of three cases. J Oral Implantol. 2008; 34(6):319-24.

14. Garg A, Funakoshi E, Misch C, Shanelec D, Tibbits L. Chapter 26: The Osteotome Technique. People's Medical Publishing House USA Ltd (PMPH); 2007. p. 381-391. [cited July 6, 2009]. Available from: Dentistry \& Oral Sciences Source.

15. Velasco Ortega E, Pérez Pérez O, Pato Mourelo J, Lorrio Castro JM, Cruz Valiño JM. La expansión ósea en la implantología oral. Av Periodon Implantol. 2008; 20(2): 95-101.

16. Komarnyckyj OG, London RM. Osteotome single-stage dental implant placement with and without sinus elevation: a clinical report. Int J Oral Maxillofac Implants. 1998; 13(6):799-804.

17. Bruschi GB, Scipioni A, Calesini G, Bruschi E. Localized management of sinus floor with simultaneous implant placement: a clinical report. Int J Oral Maxillofac Implants. 1998; 13(2):219-26.

18. Rosen PS, Summers R, Mellado JR, Salkin LM, Shanaman RH, Marks MH, Fugazzotto PA. The bone-added osteotome sinus floor elevation technique: multicenter retrospective report of consecutively treated patients. Int J Oral Maxillofac Implants. 1999; 14(6):853-8.

19. Emmerich D, Att W, Stappert C. Sinus floor elevation using osteotomes: a systematic review and meta-analysis. J Periodontol. 2005; 76(8):1237-51.

20.Sforza NM, Marzadori M, Zucchelli G. Simplified osteotome sinus augmentation technique with simultaneous implant placement: a clinical study. Int J Periodontics Restorative Dent. 2008; 28(3):291-9.

21. Nedir R, Nurdin N, SzmuklerMoncler S, Bischof $\mathrm{M}$. Osteotome sinus floor elevation technique without grafting material and immediate implant placement in atrophic posterior maxilla: report of 2 cases. J Oral Maxillofac Surg. 2009; 67(5):1098-103. 